\title{
Correction to: Adaptive Mechanisms of the Model Photosynthetic Organisms, Cyanobacteria, to Iron Deficiency
}

\author{
Hai-Bo Jiang, Xiao-Hui Lu, Bin Deng, Ling-Mei Liu, and Bao-Sheng Qiu
}

\section{Correction to: \\ Chapter 11 in: Q. Wang (ed.), Microbial Photosynthesis, https://doi.org/10.1007/978-981-15-3110-1_11}

The original version of the book was revised with the following corrections.

1. In the 10th line of Page 6, the word Fe(II)L was changed as $\mathrm{Fe}(\Pi) \mathrm{L}$.

2. In Fig. 4, the figure legend was replaced from "Miethke and Marahiel 2007" to "Shaked and Lis 2012".

3. A list of references was newly added along with its in-text citations as given below:

Trujillo, A. (2011). The iron hypothesis. http://www.homepages.ed.ac.uk/shs/ Climatechange/Carbon\%20sequestration/Martin\%20iron.htm

Moeck, G. S., \& Coulton, J. W. (1998). TonB-dependent iron acquisition: Mechanisms of siderophore-mediated active transport. Molecular Microbiology, 28(4), 675-681.

Teresa, B. M., Hernández, J. A., Luisa, P. M., et al. (2001). Cloning, overexpression and interaction of recombinant Fur from the cyanobacterium Anabaena PCC 7119 with isiB and its own promoter. FEMS Microbiology Letters, 194(2), $187-192$. 